\title{
Quantification of left atrial strain and strain rate using Cardiovascular Magnetic Resonance myocardial feature tracking: a feasibility study
}

Johannes Tammo Kowallick ${ }^{1,7}$, Shelby Kutty ${ }^{2}$, Frank Edelmann ${ }^{3,7}$, Amedeo Chiribiri ${ }^{4}$, Adriana Villa ${ }^{4}$, Michael Steinmetz ${ }^{5,7}$, Jan Martin Sohns ${ }^{1,7}$, Wieland Staab ${ }^{1,7}$, Nuno Bettencourt ${ }^{4,6}$, Christina Unterberg-Buchwald ${ }^{1,3,7}$, Gerd Hasenfuß $\beta^{3,7}$, Joachim Lotz ${ }^{1,7}$ and Andreas Schuster ${ }^{3,4,7^{*}}$

\begin{abstract}
Background: Cardiovascular Magnetic Resonance myocardial feature tracking (CMR-FT) is a quantitative technique tracking tissue voxel motion on standard steady-state free precession (SSFP) cine images to assess ventricular myocardial deformation. The importance of left atrial (LA) deformation assessment is increasingly recognized and can be assessed with echocardiographic speckle tracking. However atrial deformation quantification has never previously been demonstrated with CMR. We sought to determine the feasibility and reproducibility of CMR-FT for quantitative derivation of LA strain and strain rate (SR) myocardial mechanics.
\end{abstract}

Methods: 10 healthy volunteers, 10 patients with hypertrophic cardiomyopathy (HCM) and 10 patients with heart failure and preserved ejection fraction (HFpEF) were studied at 1.5 Tesla. LA longitudinal strain and SR parameters were derived from SSFP cine images using dedicated CMR-FT software (2D CPA MR, TomTec, Germany). LA performance was analyzed using 4- and 2-chamber views including LA reservoir function (total strain $\left[\varepsilon_{5}\right]$, peak positive SR [SRs]), LA conduit function (passive strain $\left[\varepsilon_{\mathrm{e}}\right]$, peak early negative SR [SRe]) and LA booster pump function (active strain $\left[\varepsilon_{a}\right]$, late peak negative SR [SRa]).

Results: In all subjects LA strain and SR parameters could be derived from SSFP images. There was impaired LA reservoir function in HCM and HFpEF ( $\varepsilon_{\mathrm{s}}$ [\%]: HCM 22.1 \pm 5.5 , HFpEF 16.3 \pm 5.8 , Controls $29.1 \pm 5.3, \mathrm{p}<0.01$; SRs $\left[\mathrm{s}^{-1}\right.$ ]: HCM $0.9 \pm 0.2$, HFpEF $0.8 \pm 0.3$, Controls $1.1 \pm 0.2, \mathrm{p}<0.05)$ and impaired LA conduit function as compared to healthy controls $\left(\varepsilon_{\mathrm{e}}[\%]\right.$ : HCM 10.4 \pm 3.9 , HFpEF 11.9 \pm 4.0 , Controls $21.3 \pm 5.1, \mathrm{p}<0.001$; SRe $\left[\mathrm{s}^{-1}\right]$ : HCM $-0.5 \pm 0.2$, HFpEF $-0.6 \pm 0.1$, Controls $-1.0 \pm 0.3, p<0.01$ ). LA booster pump function was increased in HCM while decreased in HFpEF ( $\varepsilon_{a}[\%]$ : HCM 11.7 $\pm 4.0, \mathrm{HFpEF} 4.5 \pm 2.9$, Controls $7.8 \pm 2.5, \mathrm{p}<0.01$; SRa [ ${ }^{-1}$ ]: HCM $-1.2 \pm 0.4, \mathrm{HFpEF}-0.5 \pm$ 0.2 , Controls $-0.9 \pm 0.3, p<0.01)$. Observer variability was excellent for all strain and SR parameters on an intra- and inter-observer level as determined by Bland-Altman, coefficient of variation and intraclass correlation coefficient analyses.

Conclusions: CMR-FT based atrial performance analysis reliably quantifies LA longitudinal strain and SR from standard SSFP cine images and discriminates between patients with impaired left ventricular relaxation and healthy controls. CMR-FT derived atrial deformation quantification seems a promising novel approach for the study of atrial performance and physiology in health and disease states.

Keywords: Cardiovascular magnetic resonance, Feature tracking, Diastolic dysfunction, Hypertrophic cardiomyopathy, Strain, Strain rate, Atrial physiology

\footnotetext{
* Correspondence: andreas_schuster@gmx.net

${ }^{3}$ Department of Cardiology and Pneumology, Georg-August-University,

Göttingen, Germany

${ }^{4}$ Division of Imaging Sciences and Biomedical Engineering, The Rayne

Institute, St. Thomas' Hospital, King's College London, London, UK

Full list of author information is available at the end of the article
} 


\section{Background}

Left atrial (LA) function is increasingly recognized to have an incremental role in determining prognosis and risk stratification in different states of disease - especially in those that are associated with ventricular diastolic dysfunction. The principal role of the LA is to modulate left ventricular filling due to three basic functional elements [1]: 1. Reservoir function (collection of pulmonary venous return during ventricular systole); 2. Conduit function (passage of blood to the left ventricle during early diastole) and 3. Contractile booster pump function (augmentation of ventricular filling during late diastole).

Echocardiographic speckle tracking has proved to be a feasible and reproducible technique to evaluate LA longitudinal strain and strain rate (SR) [2]. At the present time, the role of Cardiovascular Magnetic Resonance (CMR) to evaluate atrial function is mainly complementary to echocardiography in specific clinical instances, e.g. in diagnostic evaluation and follow-up for patients with poor echocardiographic windows [3]. CMR feature tracking (CMR-FT) - a technique analogous to echocardiographic speckle tracking - represents a novel approach to assess myocardial deformation directly from standard steady-state free precession (SSFP) cine CMR images and therefore does not require additional tagging sequence acquisitions $[4,5]$. CMR-FT makes use of offline tracking of tissue voxel motion allowing the evaluation of longitudinal, circumferential and radial myocardial deformation. The technique has been used to analyze left and right ventricular performance in health and disease [6-8]. However, the feasibility of CMR-FT for the assessment of quantitative LA function and deformation has never previously been demonstrated [9]. The aim of the present study is therefore to evaluate the feasibility and reproducibility of CMR-FT for the quantification of atrial physiology as assessed with LA strain and SR.

\section{Methods}

The study protocol was approved by the institutional review board. 10 subjects who met the conditions for HFpEF according to current consensus statements [10] (presence of signs or symptoms of congestive heart failure, presence of preserved left ventricular (LV) systolic function and echocardiographic evidence of diastolic LV dysfunction), 10 subjects with HCM (according to genetic confirmation or wall thickness $\geq 15 \mathrm{~mm}$ or $\geq 13 \mathrm{~mm}$ in case of a family history of HCM, absence of chamber dilation, absence of other systemic or cardiac disease sufficient to justify the hypertrophy) and 10 healthy controls were recruited after written informed consent was obtained. Exclusion criteria included atrial fibrillation, claustrophobia, impaired renal function, pacemaker/defibrillator devices or other metallic implants.

\section{CMR}

All CMR measurements were performed at 1.5 Tesla (Philips Intera, Philips Achieva, Siemens Sonata or Siemens Symphony TIM) in the supine position. LV dimensions and function were assessed with ECG-gated SSFP cine sequences during brief periods of breath-holding in the following planes: 12 to 14 equidistant short-axis planes covering entire ventricles as well as 2-chamber and 4chamber views. Typical image parameters were: repetition time (TR) $3.3 \mathrm{~ms}$, echo time (TE) $1.6 \mathrm{~ms}$, matrix size $228 \times 220$, field of view (FOV) $270 \times 260 \mathrm{~mm}$, slice thickness 6-8 mm (Philips Intera); TR $2.7 \mathrm{~ms}$, TE 1.3, matrix size $208 \times 184$, FOV $260 \times 230 \mathrm{~mm}$, slice thickness $8 \mathrm{~mm}$ (Philips Achieva); TR $30.9 \mathrm{~ms}$, TE $1.3 \mathrm{~ms}$, matrix size $256 \times 164$, FOV $400 \times 256 \mathrm{~mm}$, slice thickness $6-8 \mathrm{~mm}$ (Siemens Sonata); TR $45.9 \mathrm{~ms}$, TE $1.3 \mathrm{~ms}$, matrix size $192 \times 192 \mathrm{~mm}$, FOV $340 \times 340$, slice thickness $6 \mathrm{~mm}$ (Siemens Symphony TIM).

\section{Feature tracking}

LA myocardial feature tracking was performed using dedicated software (TomTec Imaging Systems, 2D CPA MR, Cardiac Performance Analysis, Version 1.1.2.36, Unterschleissheim, Germany). LA endocardial borders were manually traced in the 2- and 4-chamber views using a point-and-click approach when the atrium was at its minimum volume after atrial contraction. The atrial endocardial border surface was manually delineated and the automated tracking algorithm was applied. Tracking performance was visually reviewed to ensure accurate tracking of the atrial myocardium (Figure 1). In case of insufficient automated border tracking, manual adjustments were made to the initial contour and the algorithm was reapplied. The atrium was divided into six segments and averaged strain and strain rate profiles were calculated for all segments as previously described [11]. Tracking performance was visually reviewed on a segmental basis. If the tracking quality was not sufficient, e.g. due to the presence of pulmonary veins or left atrial appendage, the corresponding segment was excluded from the analysis. Tracking was repeated for three times in both the 2- and 4chamber view. LA longitudinal strain and SR results were averaged across all three repetitions in both views. Similar to previous definitions from echocardiographic speckle tracking $[11,12]$ three aspects of atrial strain were analysed (Figure 2): passive strain ( $\varepsilon_{\mathrm{e}}$, corresponding to atrial conduit function), active strain $\left(\varepsilon_{\mathrm{a}}\right.$, corresponding to atrial contractile booster pump function) and total strain, the sum of passive and active strain $\left(\varepsilon_{\mathrm{s}}\right.$, corresponding to atrial reservoir function). Accordingly, three SR parameters were evaluated (Figure 2): peak positive strain rate (SRs, corresponding to atrial reservoir function), peak early negative strain rate (SRe, corresponding to atrial conduit function) 

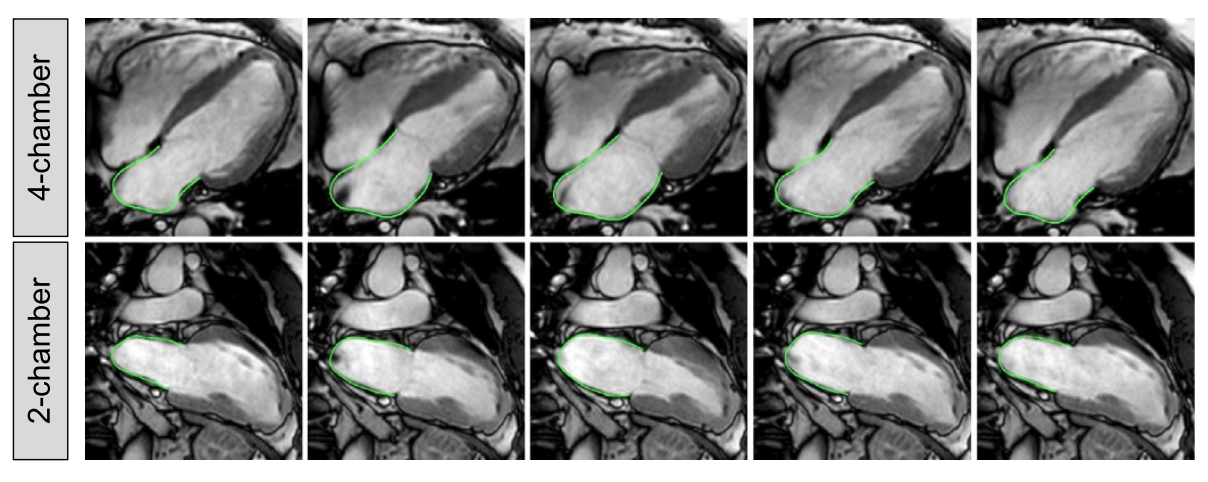

Figure 1 Left atrial CMR feature tracking. The figure shows a representative example of left atrial tracking in the 4-chamber and 2-chamber view in a patient with hypertrophic cardiomyopathy.

and peak late negative strain rate $(\mathrm{SRa}$, corresponding to atrial contractile booster pump function) $[1,13]$.

\section{Volumetric analysis}

Volumetric analysis was performed with commercially available software (QMass, Medis, Leiden, The Netherland). Left and right ventricular ejection fraction, end-diastolic and end-systolic volumes were assessed from the shortaxis stack. End-diastolic and end-systolic volumes were normalized to body surface area. Semi-automated tracings of the LA area and length were performed in the 2 - and 4-chamber view. LA volumes were calculated using the previously validated biplane area-length method [14] according to the formula: LA volume $(\mathrm{ml})=0.85^{*} \mathrm{~A}_{2 \mathrm{C}}{ }^{*} \mathrm{~A}_{4 \mathrm{C}} / \mathrm{L}$, where $\mathrm{A}_{2 \mathrm{C}}$ and $\mathrm{A}_{4 \mathrm{C}}$ are the $\mathrm{LA}$ areas on the 2-chamber and 4-chamber views,

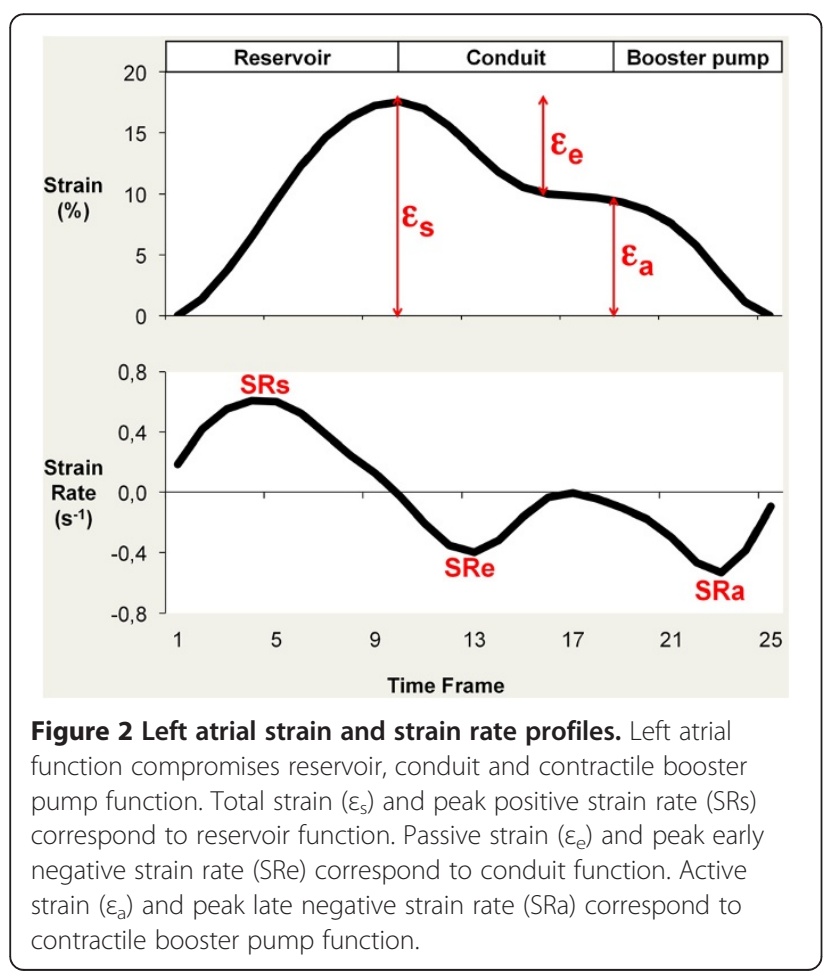

respectively, and $\mathrm{L}$ is the shorter long-axis length of the LA from either the 2-chamber or the 4-chamber views. LA volumes were assessed at left ventricular end-systole (LAVmax), at left ventricular diastole just before LA contraction (LAVpre-ac) and at late left ventricular end diastole after LA contraction (LAVmin) $[1,15]$. Total LA emptying fraction (LAEF Total, corresponding to atrial reservoir function), passive LA emptying fraction (LAEF passive, corresponding to atrial conduit function) and LA active emptying fraction (LAEF Booster, corresponding to atrial contractile booster pump function) were defined as fractional volume changes according to the following equations:

$$
\begin{aligned}
& \text { LAEF Total }=\frac{(\text { LAVmax }- \text { LAVmin }) \times 100}{L A V \max } \\
& \text { LAEF Passive }=\frac{(\text { LAVmax }- \text { LAVpre-ac }) \times 100}{L A V m a x} \\
& \text { LAEF Booster }=\frac{(\text { LAVpre }-\mathrm{ac}-\text { LAVmin }) \times 100}{\text { LAVpre-ac }}
\end{aligned}
$$

\section{Statistical analysis}

Statistical analysis was performed using Microsoft Excel and IBM SPSS Statistics version 22 for Macintosh. Data are expressed as mean ( \pm standard deviation). Differences between groups in continuous variables were assessed by the Kruskal-Wallis test. Pearson's correlation coefficients were calculated to investigate for potential relations between variables from CMR-FT and volumetric analyses regarding LA reservoir, conduit and contractile booster pump function. Correlation coefficients were considered weak if $r \leq 0.35$, moderate if $r$ was between 0.36-0.67 and strong if $r \geq 0.68$. The intra- and inter-observer variability for strain and SR measurements were assessed by the coefficient of variation $(\mathrm{CV})$, intraclass correlation coefficient (ICC) and Bland Altman analysis [16] in 10 randomly selected subjects. The CV was defined as the standard deviation of the differences divided by the mean [17]. Two independent observers analysed all cases to assess 
inter-observer variability (JTK \& AS), while intraobserver variability was derived from the repeated analysis by the first observer (JTK) after four weeks. All statistical tests with $\mathrm{p}$ values $<0.05$ were considered statistically significant.

\section{Results}

Healthy controls were younger than patients with $\mathrm{HCM}$ and HFpEF. LA volumes were higher in both patient groups than in healthy controls. Participant demographics are summarised in Table 1.

\section{Feasibility of left atrial CMR-FT}

LA CMR-FT was successfully performed in all subjects. Atrial strain profiles followed atrial physiology and allowed an evaluation of atrial total, passive and active strain. Furthermore, peak positive SR, peak early negative SR and peak late negative SR were successfully analysed in all cases. Tracking quality was sufficient in 321/360 segments (89.2\%). In 14/30 subjects, averaged strain and SR profiles were calculated from all six segments in both the 2-chamber and 4-chamber view. In 16/30 subjects, 39 individual segments were excluded (15 segments in 4 -chamber view, 24 segments in 2 -chamber view) from the analyses resulting in a total proportion of $10.8 \%$ of excluded segments. Exclusion of segments was predominantly associated with poor tracking quality related to the insertion of the pulmonary veins.

Bivariate correlation demonstrated a strong to moderate relation between volumetric indexes and deformation

Table 1 Subject characteristics

\begin{tabular}{|c|c|c|c|}
\hline & HFpEF $(n=10)$ & $\mathrm{HCM}(n=10)$ & Controls $(n=10)$ \\
\hline Gender (m/f) & $7 / 3$ & $9 / 1$ & $5 / 5$ \\
\hline Age (y) & $69.7(58-82)$ & $59.1(44-73)$ & $40.6(23-51)$ \\
\hline LV-EDV $\left(\mathrm{ml} / \mathrm{m}^{2}\right)$ & $70.6 \pm 12.2$ & $71.6 \pm 10.7$ & $76.9 \pm 12.5$ \\
\hline LV-ESV $\left(\mathrm{ml} / \mathrm{m}^{2}\right)$ & $25.8 \pm 5.4$ & $23.5 \pm 6.4$ & $33.4 \pm 7.5$ \\
\hline LVEF (\%) & $63.6 \pm 3.0$ & $67.4 \pm 6.4$ & $56.9 \pm 4.4$ \\
\hline RV-EDV $\left(\mathrm{ml} / \mathrm{m}^{2}\right)$ & $84.3 \pm 11.8$ & $71.4 \pm 11.2$ & $76.6 \pm 14.3$ \\
\hline RV-ESV $\left(\mathrm{ml} / \mathrm{m}^{2}\right)$ & $39.0 \pm 13.7$ & $24.4 \pm 6.1$ & $32.1 \pm 8.6$ \\
\hline RVEF (\%) & $54.7 \pm 8.9$ & $65.7 \pm 5.7$ & $58.5 \pm 4.1$ \\
\hline Presence of $M^{*}$ & $2(20 \%)$ & $4(40 \%)$ & $0(0 \%)$ \\
\hline LAV $\max \left(\mathrm{ml} / \mathrm{m}^{2}\right)$ & $53.1 \pm 11.2$ & $50.1 \pm 13.6$ & $34.8 \pm 9.1$ \\
\hline LAV $\min \left(\mathrm{ml} / \mathrm{m}^{2}\right)$ & $24.8 \pm 6.3$ & $20.6 \pm 7.2$ & $13.4 \pm 4.2$ \\
\hline LAV p-ac $\left(\mathrm{ml} / \mathrm{m}^{2}\right)$ & $40.1 \pm 7.9$ & $37.7 \pm 12.3$ & $23.0 \pm 7.7$ \\
\hline
\end{tabular}

Continuous variables are expressed as mean \pm standard deviation; age is expressed as median with range; $\mathrm{HFpEF}$, heart failure with preserved ejection fraction; HCM, hypertrophic cardiomyopathy; LV, left ventricle; EDV: end-diastolic volume; ESV, end-systolic volume; $E F$, ejection fraction; $m$, male; $f$, female; $y$, years; LAV max, maximum left atrial volume; LAV min, minimum left atrial volume; LAV $\mathrm{p}$-ac, left atrial volume prior to atrial contraction.

*Mitral regurgitation (MR) as defined by visual appearance of a regurgitation jet on cine SSFP. parameters from CMR-FT for atrial reservoir, conduit and contractile booster pump functions (Table 2).

\section{Left atrial function in patients and controls}

LA strain and SR parameters from CMR-FT revealed significantly different values between patients with HCM or HFpEF and healthy controls (Table 3). $\varepsilon_{\mathrm{s}}$ and SRs (both corresponding to LA reservoir function) as well as $\varepsilon_{\mathrm{e}}$ and SRe (both corresponding to LA conduit function) were lower in both patient groups. Interestingly, $\varepsilon_{\mathrm{a}}$ and SRa (both corresponding to LA booster pump function) were increased in patients with HCM while decreased in patients with HFpEF. These parameters were not significantly different in patients with mitral regurgitation as compared to patient without mitral regurgitation both in the HCM and HFpEF group.

\section{Intra- and inter-observer variability}

LA strain and SR parameters were reproducible on an intra- and inter-observer level. Bland-Altman Plots for strain and SR measurements are displayed in Figures 3 and 4, respectively. Table 4 shows ICC and CV for repeated measurements within the single and between observers.

\section{Discussion}

Our data demonstrate that LA longitudinal strain and SR parameters can be derived from CMR-FT using routinely acquired SSFP sequences in healthy volunteers, patients with HFpEF and patients with HCM. We found a good correlation between CMR-FT derived strain and SR parameters and LA volumetric indexes regarding LA reservoir, conduit and contractile booster pump function.

CMR-FT of the LA myocardium was successfully performed in all study subjects with a sufficient tracking quality in the vast majority of segments (89.2\%). Importantly the achieved tracking quality of CMR-FT is superior to the reported tracking quality of echocardiographic speckle tracking. Vianna-Pinton et al. [18] reported an exclusion rate of $6 \%$ of all participants in a group of

Table 2 Bivariate correlation of left atrial functional indexes from volumetric analysis and corresponding strain and strain rate parameters from CMR-FT

\begin{tabular}{lllll}
\hline Left atrial function & CMR-FT & $\begin{array}{l}\text { Volumetric } \\
\text { index }\end{array}$ & $\begin{array}{l}\text { Correlation } \\
\text { coefficient }\end{array}$ & P value \\
\hline Reservoir & $\varepsilon_{\mathrm{s}}$ & LAEF Total & 0.81 & $<0.001$ \\
& SRs & LAEF Total & 0.73 & $<0.001$ \\
Conduit & $\varepsilon_{\mathrm{e}}$ & LAEF Passive & 0.76 & $<0.001$ \\
& SRe & LAEF Passive & -0.82 & $<0.001$ \\
Booster pump & $\varepsilon_{\mathrm{a}}$ & LAEF Booster & 0.52 & $<0.005$ \\
& SRa & LAEF Booster & -0.63 & $<0.001$ \\
\hline
\end{tabular}

CMR-FT, Cardiovascular Magnetic Resonance myocardial feature tracking; $\varepsilon$, strain; $\mathrm{SR}$, strain rate; $\mathrm{LAEF}$, left atrial emptying fraction. 
Table 3 Comparison of left atrial volumetric indexes, strain $(\varepsilon)$ and strain rate (SR) parameters among patients with heart failure with preserved ejection fraction (HFpEF), patients with hypertrophic cardiomyopathy (HCM) and healthy controls

\begin{tabular}{|c|c|c|c|c|c|c|c|c|}
\hline & & \multirow[b]{2}{*}{$\begin{array}{l}\text { HFpEF } \\
(n=10)\end{array}$} & \multirow[b]{2}{*}{$\begin{array}{l}\text { HCM } \\
(n=10)\end{array}$} & \multirow[b]{2}{*}{$\begin{array}{l}\text { Controls } \\
(n=10)\end{array}$} & \multicolumn{4}{|l|}{$P$ value } \\
\hline & & & & & Overall & $\begin{array}{l}\text { HCM vs. } \\
\text { controls }\end{array}$ & $\begin{array}{l}\text { HCM vs. } \\
\text { HFpEF }\end{array}$ & $\begin{array}{l}\text { HFpEF vs. } \\
\text { control }\end{array}$ \\
\hline Left atrial function & $\begin{array}{l}\text { Left atrial volumetric } \\
\text { index (\%) }\end{array}$ & & & & & & & \\
\hline Reservoir & LAEF Total & $53.3(6.7)$ & $59.4(5.8)$ & $61.4(6.0)$ & 0.028 & 0.290 & 0.070 & 0.013 \\
\hline Conduit & LAEF Passive & $24.4(4.3)$ & $25.6(6.2)$ & $35.2(8.8)$ & 0.010 & 0.019 & 0.705 & 0.005 \\
\hline \multirow[t]{2}{*}{ Booster pump } & LAEF Booster & $29.0(7.3)$ & $33.8(5.7)$ & $26.2(6.8)$ & 0.041 & 0.013 & 0.122 & 0.406 \\
\hline & Left atrial strain (\%) & & & & & & & \\
\hline Reservoir & $\varepsilon_{\mathrm{s}}$ & $16.3(5.8)$ & $22.1(5.5)$ & $29.1(5.3)$ & 0.001 & 0.008 & 0.034 & 0.001 \\
\hline Conduit & $\varepsilon_{\mathrm{e}}$ & $11.9(4.0)$ & $10.4(3.9)$ & $21.3(5.1)$ & $<0.001$ & $<0.001$ & 0.650 & 0.001 \\
\hline \multirow[t]{2}{*}{ Booster pump } & $\varepsilon_{\mathrm{a}}$ & $4.5(2.9)$ & $11.7(4.0)$ & $7.8(2.5)$ & 0.001 & 0.023 & 0.001 & 0.019 \\
\hline & Left atrial strain rate $\left(\mathrm{s}^{-1}\right)$ & & & & & & & \\
\hline Reservoir & SRs & $0.8(0.3)$ & $0.9(0.2)$ & $1.1(0.2)$ & 0.023 & 0.028 & 0.384 & 0.017 \\
\hline Conduit & SRe & $-0.6(0.1)$ & $-0.5(0.2)$ & $-1.0(0.3)$ & 0.002 & 0.002 & 0.733 & 0.003 \\
\hline \multirow[t]{5}{*}{ Booster pump } & SRa & $-0.5(0.2)$ & $-1.2(0.4)$ & $-0.9(0.3)$ & 0.004 & 0.131 & 0.004 & 0.010 \\
\hline & Left atrial volume $\left(\mathrm{ml} / \mathrm{m}^{2}\right)$ & & & & & & & \\
\hline & LAV max & $53.1(11.2)$ & $50.1(13.6)$ & $34.8(9.1)$ & 0.004 & 0.013 & 0.623 & 0.002 \\
\hline & LAV min & $24.8(6.3)$ & $20.6(7.2)$ & $13.4(4.2)$ & 0.002 & 0.021 & 0.186 & 0.001 \\
\hline & LAV p-ac & 40.1 (7.9) & $37.7(12.3)$ & $23.0(7.7)$ & 0.002 & 0.011 & 0.597 & 0.001 \\
\hline
\end{tabular}

$\mathrm{HFpEF}$, heart failure with preserved ejection fraction; HCM, hypertrophic cardiomyopathy; LAEF, left atrial emptying fraction; $\varepsilon$, strain; SR, strain rate; LAV max, maximum left atrial volume; LAV min, minimum left atrial volume; LAV p-ac, left atrial volume prior to atrial contraction. Bold $\mathrm{p}$ values indicate a significance level $<0.05$.

healthy volunteers in their study using echocardiographic speckle tracking based atrial performance analysis. In the remaining study cohort, speckle tracking quality was sufficient in $91 \%$ of all segments. The technique used in this work represents the current echocardiographic standard for LA speckle tracking analysis as recommended in current guidelines [19].

The current reference standard for quantitative wall motion assessment with CMR is myocardial tagging. Myocardial tagging based strain assessment remains time-consuming since additional sequences and complex post processing are required. In the context of LA function assessment, the main problem with myocardial tagging is its limitation to ventricular deformation quantification [20]. The LA myocardium appears to be not thick enough to be labeled by grid lines. Displacement encoding with stimulated echoes (DENSE) represents a further technique that allows for quantitative assessment of myocardial deformation. Again, DENSE has not proved to be feasible in the assessment of atrial strain and SR. However, DENSE might contain a potential to assess deformation of narrow anatomical structures (e.g. the LA myocardium) since recent data indicate the feasibility to quantify aortic wall stretch [21]. In essence, CMR-FT represents the only CMR based technique that allows for LA strain and SR imaging at the present time.

\section{Left atrial function in patients with HCM and HFpEF}

Abnormal LA function is present in patients with ventricular diastolic dysfunction. For instance, Kaminski et al. demonstrated that impaired LA contractile function was the strongest predictor of major adverse cardiac events and all-cause mortality in patients with chronic hypertension but no other prevalent cardiovascular disease [22]. Since diastolic dysfunction is present in HFpEF and HCM as well $[23,24]$ it is particularly interesting to investigate LA deformation in both patient groups. Looking at our data, CMR-FT revealed significantly decreased passive strain and peak early negative SR in patients with HCM and HFpEF. Both parameters correspond to atrial conduit function. LA conduit function is closely related to left ventricular compliance [1]. Therefore, decreased passive strain and decreased peak early negative SR from CMRFT might be directly correlated with impaired ventricular relaxation. Furthermore, total strain and peak positive SR correspond to atrial reservoir function. Both parameters were decreased in HCM and HFpEF, which might refer to impaired atrial compliance. Increases in booster pump function in HCM have previously been reported $[1,25,26]$. 


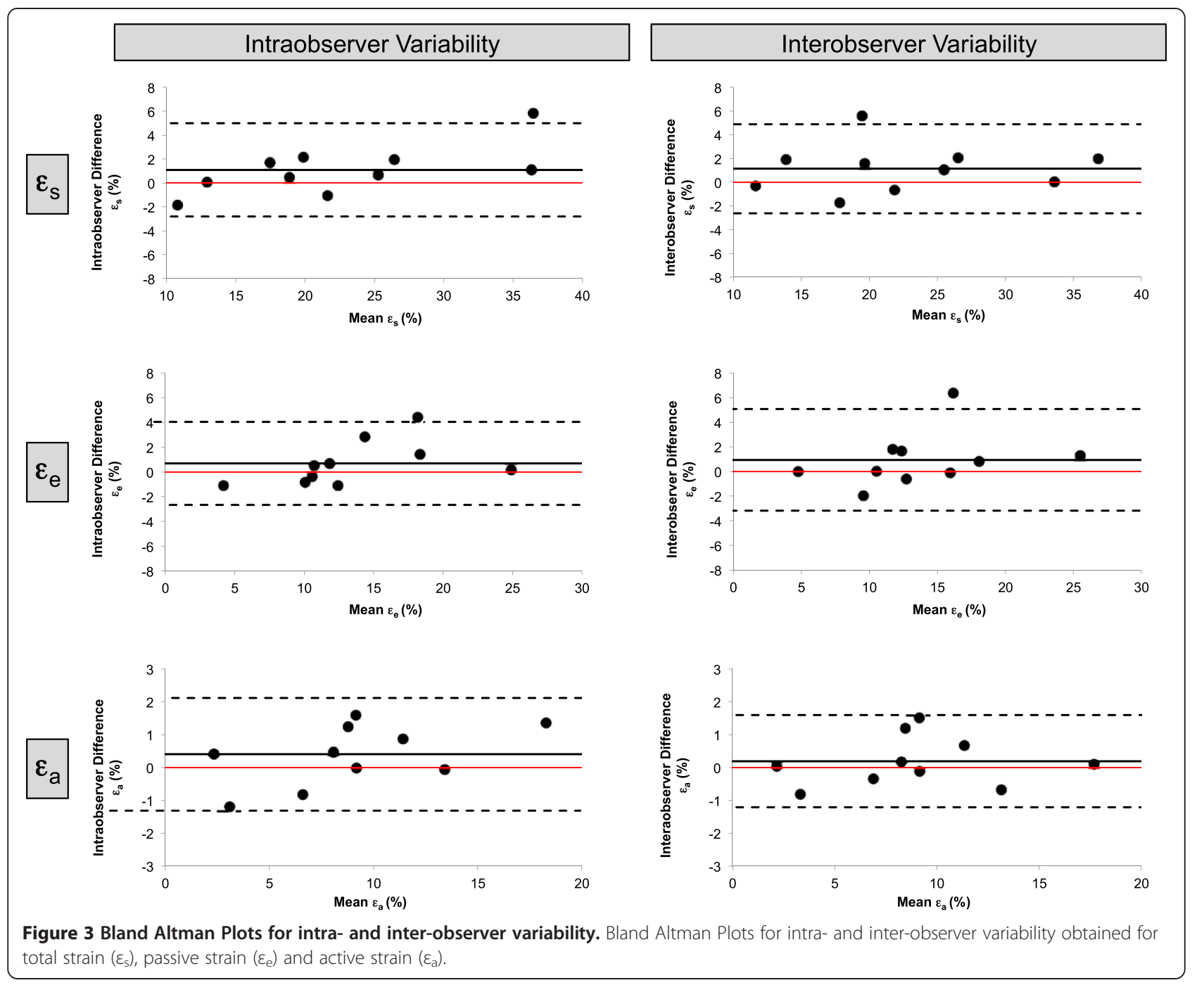

In contrast, impaired atrial contractility has been described in HFpEF by echocardiographic speckle tracking [27]. Consistently, our results show increased active strain and peak late negative SR (both corresponding to contractile booster pump function) in HCM while decreased in HFpEF. The presence of mitral regurgitation did not seem to be associated with the increased booster pump function in the HCM group. It might be interesting to speculate whether or not the increased booster pump function with decreased reservoir and conduit function in HCM represents a form of compensated milder functional diastolic dysfunction as opposed to severe diastolic dysfunction in HFpEF with complete atrial performance impairment. Indeed Murata et al. reported initial increase in LA booster pump function at early stages of impaired left ventricular relaxation, followed by progressive decompensation of global LA performance with worsening of diastolic dysfunction at later disease stages [28]. Future studies will need to investigate whether atrial performance analysis could be a useful parameter for the identification of patients at risk for congestive heart failure with associated poor prognosis that may specifically benefit from early therapeutic interventions.

\section{Reproducibility}

The reported amount of reproducibility and repeatability of ventricular strain and SR measurements from CMRFT varies between studies with most studies reporting reasonable reproducibility of global strain and SR levels [5,29-31]. Therefore, intra- and inter-observer variability of LA deformation indexes need to be taken into special consideration. In order to further maximise reproducibility all measurements were repeated three times in both the 2-chamber and 4-chamber view. All strain and SR parameters had excellent reproducibility both on the intra-observer as well as inter-observer level. The calculation of global strain and SR parameters (average of 6 segments) might explain the excellent reproducibility as 


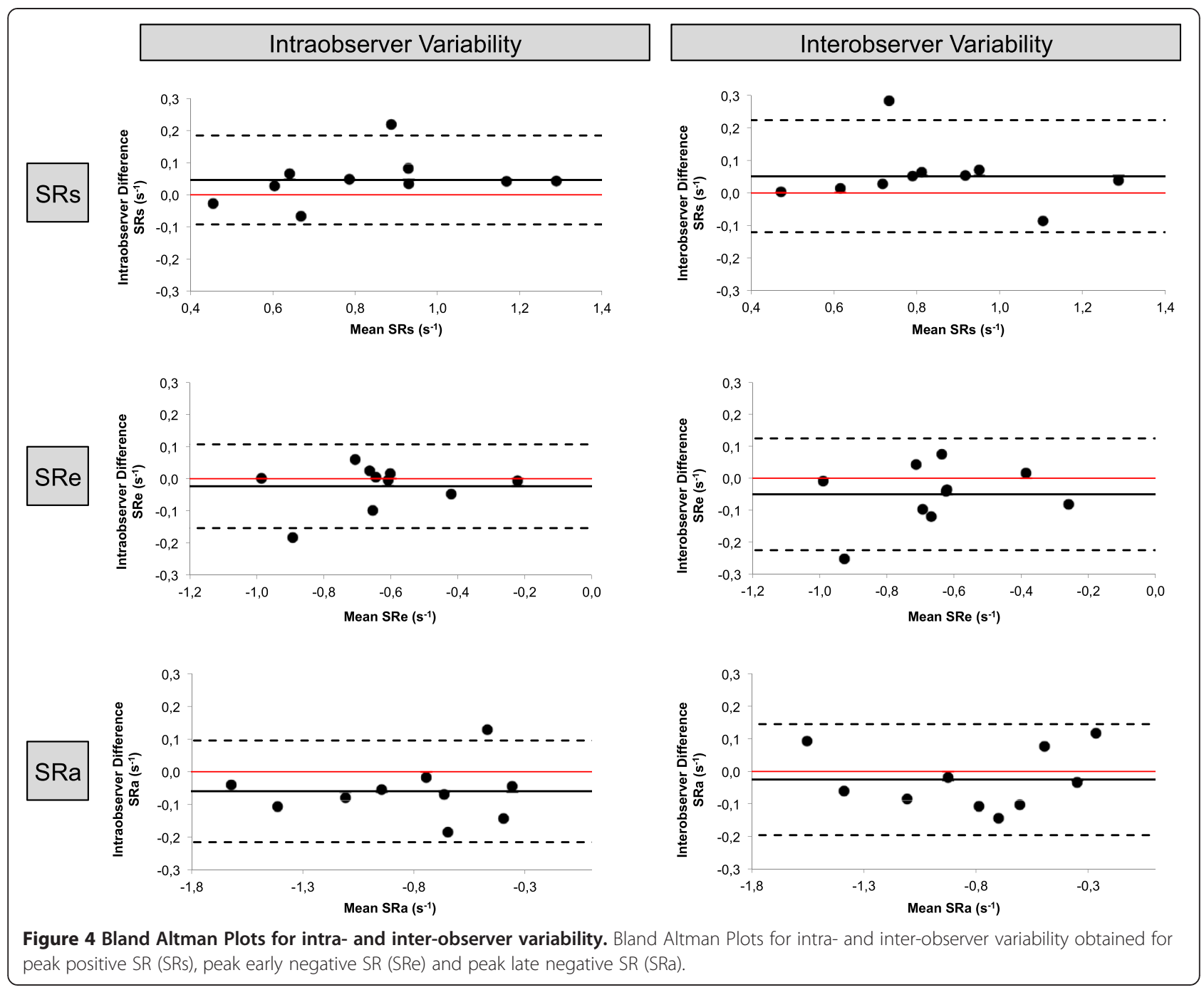

opposed to previously reported bad segmental reproducibility of some of the ventricular CMR-FT measurements [32]. Furthermore the good reproducibility might also be associated with the good image quality of the CMR images with reliable delineation of the thin LA myocardium in contrast to LA echocardiographic speckle tracking that suffer from the far-field location of the LA and reduced signal-to-noise ratio. On the other side, lower temporal resolution of CMR images might affect deformation analysis, e.g. the capacity to measure peak strain rates. However a reasonable inter-modality agreement for left ventricular longitudinal strain analysis has been demonstrated between CMR-FT and echocardiographic speckle tracking despite differences in temporal

Table 4 Intra-observer and inter-observer reproducibility for strain $(\varepsilon)$ and strain rate (SR) parameters

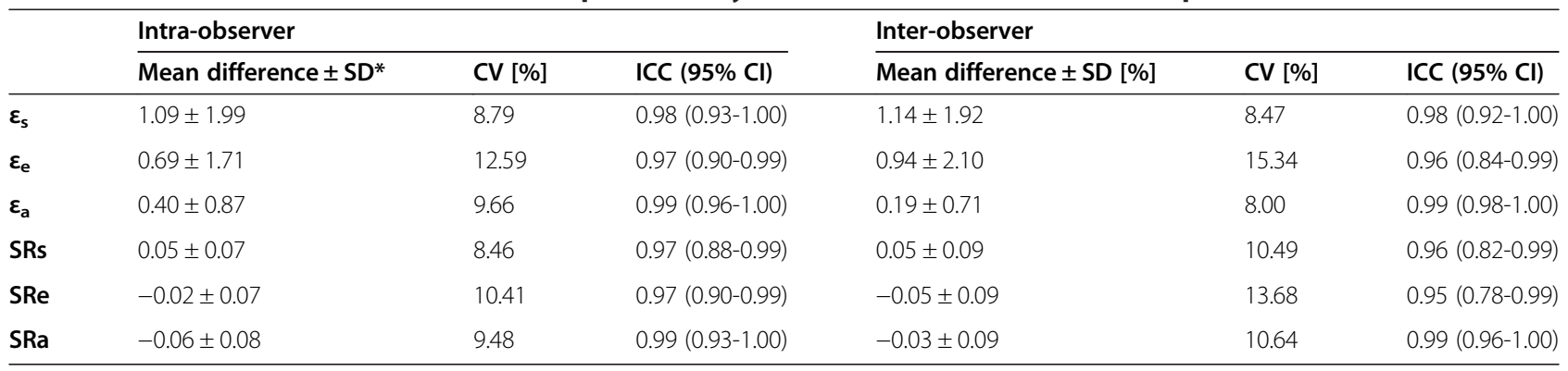

$\mathrm{SD}$, standard deviation; $\mathrm{CV}$, coefficient of variation; ICC, intraclass correlation coefficient; $\varepsilon$, strain; $\mathrm{SR}$, strain rate; ${ }^{*}$ Mean difference $\pm S D$ are given as [\%] for $\varepsilon$ and as $\left[s^{-1}\right]$ for SR parameters. 
resolution [33]. Future studies will need to investigate the effect of these parameters for LA strain and SR imaging with CMR-FT.

\section{Limitations}

We have performed a feasibility study to demonstrate the use of CMR-FT for the assessment of atrial deformation. The sample size of healthy volunteers and patients was relatively small and has not been powered to detect gross differences between groups. Nevertheless we were able to detect significant differences between health and disease and future studies will need to investigate the clinical utility of CMR-FT derived LA strain and SR parameters in a larger cohort of patients with different pathologies. CMR measurements were performed on different scanner types. Importantly the reproducibility assessed in the current study was excellent for all parameters irrespective of scanner type. This is in line with previous studies demonstrating similar reproducibility of CMR-FT ventricular strain analysis at 1.5 and 3 Tesla [30,31].

Furthermore LA CMR-FT faces several challenges as opposed to ventricular deformation tracking that may limit the performance of the LA tracking: 1 . The LA myocardial wall is thinner than the ventricular myocardial wall, 2. LA anatomy is more variable than ventricular anatomy, 3. LA physiology is quite complex (reservoir function, conduit function, booster pump function), 4. The presence of pulmonary veins and left atrial appendage can compromise tracking quality. Notwithstanding these facts, CMR-FT analysis was successful in all controls and patients. Tracking quality was sufficient in the vast majority of segments (89.2\%) and reproducibility was excellent.

\section{Conclusions}

CMR-FT allows derivation of LA longitudinal deformation mechanics directly from standard SSFP cine images. LA strain and SR parameters were highly reproducible on an intra- and inter-observer basis. Differences in strain and SR parameters between healthy controls and patients with HCM or HFpEF were in consistence with published literature. LA CMR myocardial deformation analysis with feature tracking may have potential clinical and research applications. Further studies in larger cohorts should follow to establish the clinical utility and the prognostic implications of this technique.

\section{Abbreviations}

CMR: Cardiovascular Magnetic Resonance; CMR-FT: CMR feature tracking: CV: Coefficient of variation; $\varepsilon$ : Strain; FOV: Field of view; HCM: Hypertrophic cardiomyopathy; HFpEF: Heart failure with preserved ejection fraction; ICC: Intraclass correlation coefficient; LA: Left atrial; LAEF: Left atrial emptying fraction; LAV: Left atrial volume; SR: Strain rate; SSFP: Steady-state free precession; TE: Echo time; TR: Repetition time.

\section{Competing interests}

The authors declare that they have no competing interests.

\section{Authors' contributions}

JTK and AS designed the study protocol, performed data acquisition and measurements, performed statistical analysis and drafted the manuscript. FE, $\mathrm{GH}, J$ revised the manuscript, participated in the scientific discussion during the study. SK, AC, AV, MS, JMS, WS, NB, CUB revised the manuscript and performed data acquisition. All authors read and approved the final manuscript.

\section{Acknowledgements}

A. Schuster was funded by the Research program of the Faculty of Medicine of the Georg-August-University in Göttingen. We are grateful for the financial support provided by the DZHK (German Centre for Cardiovascular Research).

\section{Author details}

${ }^{1}$ Institute for Diagnostic and Interventional Radiology,

Georg-August-University, Göttingen, Germany. ${ }^{2}$ Children's Hospital and Medical Center Joint Division of Pediatric Cardiology, University of Nebraska/ Creighton University, Omaha, Nebraska. ${ }^{3}$ Department of Cardiology and Pneumology, Georg-August-University, Göttingen, Germany. ${ }^{4}$ Division of Imaging Sciences and Biomedical Engineering, The Rayne Institute, St. Thomas' Hospital, King's College London, London, UK. ${ }^{5}$ Department of Pediatric Cardiology and Intensive Care Medicine, Georg-August-University, Göttingen, Germany. ${ }^{6}$ Cardiology Department, Centro Hospitalar de Gaia/ Espinho, Vila Nova de Gaia, Portugal. DZHK (German Centre for Cardiovascular Research), partner site Göttingen, Göttingen, Germany.

Received: 8 May 2014 Accepted: 28 July 2014

Published: 12 August 2014

\section{References}

1. Hoit BD. Left atrial size and function: role in prognosis. J Am Coll Cardiol. 2014; 63:493-505.

2. Vieira MJ, Teixeira R, Goncalves L, Gersh BJ. Left atrial mechanics: echocardiographic assessment and clinical implications. J Am Soc Echocardiogr. 2014; 27:463-78.

3. To AC, Flamm SD, Marwick TH, Klein AL. Clinical utility of multimodality LA imaging: assessment of size, function, and structure. JACC CardiovasC Imaging. 2011; 4:788-98.

4. Kowallick JT, Edelmann F, Lotz J, Lamata P, Schuster A. Imaging diastolic dysfunction with cardiovascular magnetic resonance. J Cardiol Ther. 2014; 1:58-64.

5. Schuster A, Kutty S, Padiyath A, Parish V, Gribben P, Danford DA, Makowski MR, Bigalke B, Beerbaum P, Nagel E. Cardiovascular magnetic resonance myocardial feature tracking detects quantitative wall motion during dobutamine stress. J Cardiovasc Magn Reson. 2011; 13:58.

6. Morton G, Schuster A, Jogiya R, Kutty S, Beerbaum P, Nagel E. Inter-study reproducibility of cardiovascular magnetic resonance myocardial feature tracking. J Cardiovasc Magn Reson. 2012; 14:43.

7. Onishi T, Saha SK, Ludwig DR, Marek JJ, Cavalcante JL, Schelbert EB, Schwartzman D, Gorcsan J 3rd. Feature tracking measurement of dyssynchrony from cardiovascular magnetic resonance cine acquisitions: comparison with echocardiographic speckle tracking. J Cardiovasc Magn Reson. 2013; 15:95

8. Schuster A, Paul M, Bettencourt N, Morton G, Chiribiri A, Ishida M, Hussain S, Jogiya R, Kutty S, Bigalke B, Perera D, Nagel E. Cardiovascular magnetic resonance myocardial feature tracking for quantitative viability assessment in ischemic cardiomyopathy. Int J Cardiol. 2013; 166:413-20.

9. Hoit BD. Reply to letter to the editor: feature tracking cardiac magnetic resonance imaging in the assessment of left atrial function. J Am Coll Cardiol. 2014; 63:2435-36.

10. Paulus WJ, Tschope C, Sanderson JE, Rusconi C, Flachskampf FA Rademakers FE, Marino P, Smiseth OA, De Keulenaer G, Leite-Moreira AF, Borbély A, Edes I, Handoko ML, Heymans S, Pezzali N, Pieske B, Dickstein K, Fraser AG, Brutsaert DL. How to diagnose diastolic heart failure: a consensus statement on the diagnosis of heart failure with normal left ventricular ejection fraction by the Heart Failure and Echocardiography Associations of the European Society of Cardiology. Eur Heart J. 2007; 28:2539-50. 
11. Ring L, Rana BS, Wells FC, Kydd AC, Dutka DP. Atrial function as a guide to timing of intervention in mitral valve prolapse with mitral regurgitation. JACC Cardiovasc Imaging. 2014; 7:225-32.

12. Cameli M, Caputo M, Mondillo S, Ballo P, Palmerini E, Lisi M, Marino E, Galderisi M. Feasibility and reference values of left atrial longitudinal strain imaging by two-dimensional speckle tracking. Cardiovasc Ultrasound. 2009; 7:6

13. Kutty S, Padiyath A, Li L, Peng Q, Rangamani S, Schuster A, Danford DA. Functional maturation of left and right atrial systolic and diastolic performance in infants, children, and adolescents. J Am Soc Echocardiogr. 2013; 26:398-409. e392.

14. Sievers B, Kirchberg S, Addo M, Bakan A, Brandts B, Trappe HJ. Assessment of left atrial volumes in sinus rhythm and atrial fibrillation using the biplane area-length method and cardiovascular magnetic resonance imaging with TrueFISP. J Cardiovasc Magn Reson. 2004; 6:855-63.

15. Jarvinen $V$, Kupari $M$, Hekali $P$, Poutanen VP. Assessment of left atrial volumes and phasic function using cine magnetic resonance imaging in normal subjects. Am J Cardiol. 1994; 73:1135-38.

16. Bland JM, Altman DG. Statistical methods for assessing agreement between two methods of clinical measurement. Lancet. 1986; 1:307-10.

17. Grothues F, Smith GC, Moon JC, Bellenger NG, Collins P, Klein HU, Pennell DJ. Comparison of interstudy reproducibility of cardiovascular magnetic resonance with two-dimensional echocardiography in normal subjects and in patients with heart failure or left ventricular hypertrophy. Am J Cardiol. 2002; 90:29-34.

18. Vianna-Pinton R, Moreno CA, Baxter CM, Lee KS, Tsang TS, Appleton CP. Two-dimensional speckle-tracking echocardiography of the left atrium: feasibility and regional contraction and relaxation differences in normal subjects. J Am Soc Echocardiogr. 2009; 22:299-305.

19. Mor-Avi V, Lang RM, Badano LP, Belohlavek M, Cardim NM, Derumeaux G, Galderisi M, Marwick T, Nagueh SF, Sengupta PP, Sicari R, Smiseth OA, Smulevitz B, Takeuchi M, Thomas JD, Vannan M, Voigt JU, Zamorano JL. Current and evolving echocardiographic techniques for the quantitative evaluation of cardiac mechanics: ASE/EAE consensus statement on methodology and indications endorsed by the Japanese Society of Echocardiography. J Am Soc Echocardiogr. 2011; 24:277-313.

20. Attili AK, Schuster A, Nagel E, Reiber JH, van der Geest RJ. Quantification in cardiac MRI: advances in image acquisition and processing. Int J Cardiovasc Imaging. 2010; 26(Suppl 1):27-40.

21. Haraldsson H, Hope M, Acevedo-Bolton G, Tseng E, Zhong X, Epstein FH, Ge $L$, Saloner D. Feasibility of asymmetric stretch assessment in the ascending aortic wall with DENSE cardiovascular magnetic resonance. J Cardiovasc Magn Reson. 2014; 16:6.

22. Kaminski M, Steel K, Jerosch-Herold M, Khin M, Tsang S, Hauser T, Kwong RY. Strong cardiovascular prognostic implication of quantitative left atrial contractile function assessed by cardiac magnetic resonance imaging in patients with chronic hypertension. J Cardiovasc Magn Reson. 2011; 13:42.

23. Kurt M, Wang J, Torre-Amione G, Nagueh SF. Left atrial function in diastolic heart failure. Circ Cardiovasc Imaging. 2009; 2:10-5.

24. D'Andrea A, De Corato G, Scarafile R, Romano S, Reigler L, Mita C, Allocca F, Limongelli G, Gigantino G, Liccardo B, Cuomo S, Tagliamonte G, Caso P, Calbrò R. Left atrial myocardial function in either physiological or pathological left ventricular hypertrophy: a two-dimensional speckle strain study. Br J Sports Med. 2008; 42:696-702.

25. Anwar AM, Soliman OI, Geleijnse ML, Michels M, Vletter WB, Nemes A, ten Cate FJ. Assessment of left atrial ejection force in hypertrophic cardiomyopathy using real-time three-dimensional echocardiography. J Am Soc Echocardiogr. 2007; 20:744-48.

26. Anwar AM, Soliman OI, Nemes A, Geleijnse ML, ten Cate FJ. An integrated approach to determine left atrial volume, mass and function in hypertrophic cardiomyopathy by two-dimensional echocardiography. Int J Cardiovasc Imaging. 2008; 24:45-52.

27. Morris DA, Gailani M, Vaz Perez A, Blaschke F, Dietz R, Haverkamp W, Ozcelik C. Left atrial systolic and diastolic dysfunction in heart failure with normal left ventricular ejection fraction. J Am Soc Echocardiogr. 2011; 24:651-62.

28. Murata M, Iwanaga S, Tamura Y, Kondo M, Kouyama K, Ogawa S. A real-time three-dimensional echocardiographic quantitative analysis of left atrial function in left ventricular diastolic dysfunction. Am J Cardiol. 2008; 102:1097-102.
29. Hor KN, Gottliebson WM, Carson C, Wash E, Cnota J, Fleck R, Wansapura J, Klimeczek P, Al-Khalidi HR, Chung ES, Benson DW, Mazur W. Comparison of magnetic resonance feature tracking for strain calculation with harmonic phase imaging analysis. JACC Cardiovasc Imaging. 2010; 3:144-51.

30. Schuster A, Morton G, Hussain ST, Jogiya R, Kutty S, Asrress KN, Makowski MR, Bigalke B, Perera D, Beerbaum P, Nagel E. The intra-observer reproducibility of cardiovascular magnetic resonance myocardial feature tracking strain assessment is independent of field strength. Eur J Radiol. 2013; 82:296-301.

31. Singh A, Steadman CD, Khan JN, Horsfield MA, Bekele S, Nazir SA, Kanagala $P$, Masca NG, Clarysse P, McCann GP. Intertechnique agreement and interstudy reproducibility of strain and diastolic strain rate at 1.5 and 3 tesla: a comparison of feature-tracking and tagging in patients with aortic stenosis. J Magn Reson Imaging. 2014. doi:10.1002/jmri.24625.

32. Wu L, Germans T, Guclu A, Heymans MW, Allaart CP, van Rossum AC. Feature tracking compared with tissue tagging measurements of segmental strain by cardiovascular magnetic resonance. J Cardiovasc Magn Reson. 2014; 16:10.

33. Padiyath A, Gribben P, Abraham JR, Li L, Rangamani S, Schuster A, Danford DA, Pedrizzetti G, Kutty S. Echocardiography and cardiac magnetic resonance-based feature tracking in the assessment of myocardial mechanics in tetralogy of Fallot: an intermodality comparison. Echocardiography. 2013; 30:203-10.

\section{doi:10.1186/s12968-014-0060-6}

Cite this article as: Kowallick et al:: Quantification of left atrial strain and strain rate using Cardiovascular Magnetic Resonance myocardial feature tracking: a feasibility study. Journal of Cardiovascular Magnetic Resonance 2014 16:60.

\section{Submit your next manuscript to BioMed Central and take full advantage of:}

- Convenient online submission

- Thorough peer review

- No space constraints or color figure charges

- Immediate publication on acceptance

- Inclusion in PubMed, CAS, Scopus and Google Scholar

- Research which is freely available for redistribution 